\title{
Zonulin: A Potential Marker of Intestine Injury in Newborns
}

\author{
Anna Tarko, ${ }_{1}^{1}$ Anna Suchojad, ${ }^{1}$ Marta Michalec, ${ }^{1}$ Malgorzata Majcherczyk, ${ }^{1}$ \\ Aniceta Brzozowska, ${ }^{2}$ and Iwona Maruniak-Chudek ${ }^{1}$ \\ ${ }^{1}$ Department of Intensive Care and Neonatal Pathology, School of Medicine in Katowice, Medical University of Silesia, \\ Katowice, Poland \\ ${ }^{2}$ Health Promotion and Obesity Management Unit, Department of Pathophysiology, Faculty of Medicine in Katowice, \\ Medical University of Silesia, Katowice, Poland
}

Correspondence should be addressed to Iwona Maruniak-Chudek; ich@mp.pl

Received 7 April 2017; Accepted 7 June 2017; Published 9 July 2017

Academic Editor: Natacha Turck

Copyright (C) 2017 Anna Tarko et al. This is an open access article distributed under the Creative Commons Attribution License, which permits unrestricted use, distribution, and reproduction in any medium, provided the original work is properly cited.

Introduction. Zonulin ( $\mathrm{ZO})$, a new diagnostic biomarker of intestinal permeability, was tested in newborns presenting symptoms of infection and/or inflammation of the gut or being at risk of intestinal pathology. Material and Methods. Serum ZO was assessed in 81 newborns diagnosed with sepsis, necrotizing enterocolitis (NEC), rotavirus infection, and gastroschisis, also in extremely low gestational age babies, and in controls (healthy newborns). ZO concentration was compared to C-reactive protein (CRP) and procalcitonin (PCT) values, leucocyte and platelet count, basic demographic data, and the value of the Neonatal Therapeutic Intervention Scoring System (NTISS). Results. Median values of ZO were markedly higher in groups with rotavirus infection and gastroschisis (36.0 (1-3Q: 26.0-43.2) and 20.3 (1-3Q: 17.7-28.2) ng/ml, resp.) versus controls (3.5 (1-3Q: 2.7-4.8) ng/ml). Its concentration in the NEC group was twice as high as in controls but did not reach statistical significance. ZO levels were not related to NTISS, CRP, and PCT. Conclusions. Zonulin is a promising biomarker of intestinal condition, markedly elevated in rotavirus infections. Its role in defining the severity of necrotizing enterocolitis and the risk for perforation is not well described and needs further evaluation. An increase in zonulin may not be parallel to the release of inflammatory markers, and low CRP should not exclude an injury to neonatal intestine.

\section{Introduction}

Gastrointestinal tract is an important organ which is attributed not only to digestion and absorption of nutritional compounds but also to immunologic function, modulation of inflammatory response, and triggering of autoimmune diseases. Complexity of morphological structure of the gut is not completely revealed, and new findings show the broader meaning and significance of this organ. Particularly, in neonatal period and infancy, we are still discovering the importance of the gut in overall wellbeing. Newborns and infants are undergoing developmental changes and processes, including immunity and managing inflammatory response. The less mature newborn the more fragile tissues of gastrointestinal track appear to be predominantly in extremely low gestational age premature infants. Additionally, some illnesses may have particularly severe clinical course in this period of life, such as necrotizing enterocolitis (NEC) or sepsis (especially caused by Gram-negative species). They are both known for their poor outcome in most of the cases, and early symptoms are not specific and do not indicate prognosis (including intestinal perforation or septic shock with multiorgan dysfunction). Also, some frequent and widespread illnesses like rotavirus infection, in very rare cases, are followed by bacterial sepsis, possibly related to intestinal bacterial translocation. A search for a sensitive biomarker of intestine injury is ongoing, as the traditional parameters of inflammatory status are not sufficiently predictable.

Zonulin (ZO), as an established indicator of intestinal permeability, has been described more than 20 years ago $[1,2]$. It is claimed to have a potential value in delineating the mechanism of paracellular "gateway" in the intestines [3-5]. This $47 \mathrm{kDa}$ single protein is isolated from a membrane complex (claudin-occludin-guanylate kinase-like 
proteins $\mathrm{ZO}-1, \mathrm{ZO}-2$, and $\mathrm{ZO}-3$ ) forming tight junction (TJ) in the apical part of intestinal endothelium [6]. ZO has also been known as pre-HP2, due to its identity to the precursor of haptoglobin-2 [7]. It is one of the main factors securing the adequate action of the "gate of the gut" mechanism by reversibly influencing TJ's tightness $[8,9]$. Incorporation of proteinase-activating receptor 2 (PAR2) and epidermal growth factor receptor (EGFR) allow to cross the intestinal barrier for molecules exceeding approx. $3.5 \mathrm{kDa}$ [7]. Experimental studies revealed that the rise in $\mathrm{ZO}$ concentration is parallel to increased intestinal permeability [6]. Further, clinical investigations showed that patients suffering from entities known for component of low-grade inflammation (such as type 2 diabetes, celiac disease, and obesity) presented increased $\mathrm{ZO}$ concentration [10-13]. The same is true for diseases of autoimmunological pathomechanism (Crohn's disease, type 1 diabetes) $[6,14]$. ZO increase and disruption of $\mathrm{TJ}$ has also been demonstrated in an animal model of NEC with intestinal TJ destruction proven by immunohistochemical evaluation [15].

Based on this findings, we decided to measure serum $\mathrm{ZO}$ concentration in newborns suffering from entities typical for this population, focusing on prematurity, NEC, sepsis, and rotavirus infection. The aim of our pilot study was to check if there is any correlation between $\mathrm{ZO}$ level as a marker of intestinal permeability and concentration of inflammatory markers and also the general clinical condition of our study group.

\section{Material and Methods}

2.1. Patients. 81 newborns admitted to Intensive Care and Neonatal Pathology Department of paediatric university hospital were enrolled to this observational study. All of the patients were outborn, referred to the unit from first, second, or third level nurseries because of diagnosed pathological symptoms, or they were admitted from home through the hospital emergency unit due to sudden illness. The patients were diagnosed and treated according to the established ward protocols, and this retrospective project did not interfere with the course of medical management. Demographic and clinical data were achieved from patients' files, and ZO evaluation was performed using serum samples saved after planned laboratory tests. Severity of clinical condition and intensity of medical management were evaluated using the Neonatal Therapeutic Intervention Scoring System (NTISS).

Bioethical Committee of Medical University of Silesia gave its consent for using the remaining biological material after standard workup. The control group $(N=14)$ consisted of newborns not diagnosed finally with infection or any other condition causing inflammatory process. The sepsis group included 12 term newborns suffering from early to late severe sepsis. They were seriously ill, suffering from respiratory and, in some cases, circulatory insufficiency, treated with at least two broad-spectrum antibiotics and in 4 cases receiving catecholamines in intravenous infusions. The ELGAN group consisted of extremely low gestational age newborns (ELGAN) on their first days of life, hospitalized for suspected intrauterine infection, requiring mechanical ventilation, total parenteral nutrition (TPN), and constant monitoring of basic biophysical parameters. The NEC group included 2 term, 2 near-term, and 8 preterm babies diagnosed with necrotising enterocolitis. In 5 cases, perforation occurred during the course of an illness. They were all intensively treated with respiratory and circulatory support, antibiotics, and TPN. In term babies and one late preterm, the occurrence of NEC was combined with congenital heart malformations causing poor peripheral perfusion. The other late preterm baby presented symptoms of NEC in the course of severe sepsis. The rotavirus group was formed of term newborns who were diagnosed with rotavirus infections. General clinical condition was stable; they did not require any respiratory or circulatory support and were enterally fed and given additionally intravenous infusion. The last group, abdominal wall defect (AWD), included only 3 patients who were diagnosed with gastroschisis and prepared for surgical closure. All serum samples were analyzed only once in each patient, focusing on the day of presenting clear clinical symptoms of the disease.

2.2. Laboratory Measurements. Serum zonulin measurements were performed using a commercially available ELISA kit (Immunodiagnostic AG, Bensheim, Germany). Mean intracoefficient of variance was less than $5 \%$, and sensitivity specified below $0.01 \mathrm{ng} / \mathrm{ml}$.

2.3. Data Analysis. Sepsis was defined as systemic inflammatory response syndrome (SIRS) with evidence of infection (positive microbiological culture, clinical symptoms). Severe sepsis was identified based on clinical and laboratory parameters with symptoms of at least two organ or system dysfunctions.

Routinely used immunological test detecting rotavirus antigen in a stool was used for the diagnosis of rotavirus infection in symptomatic newborns.

NEC was recognized based on radiologic and ultrasound findings in newborns with risk factors, presenting signs and symptoms suggesting this entity. Perforation was confirmed by X-ray imaging, and patients were qualified for surgery.

In all cases of gastroschisis, the patients were operated in a one-stage manner (underwent primary closure). Serum samples acquired from the last mentioned group were secured before the surgery.

2.4. Statistical Analysis. Analyses were performed using the STATISTICA 10.0 software (StatSoft, Tulsa, OK, U.S.). Normality of distribution was tested with the KolmogorovSmirnov test. The data presented are median values with 1 and 3 quartiles (1-3Q) due to nonnormal distribution of the variables. For comparison of groups, we used the $\chi^{2}$ test (qualitative variables) and ANOVA or Kruskal-Wallis one-way analysis of variance by ranks followed by the Mann-Whitney $U$ test (quantitative variables). Correlation coefficients were calculated according to Spearman. $p$ values $<0.05$ were considered as statistically significant. 
TABle 1: Demographic and clinical characteristics of the study group $(N=81)$ with distribution according to clinical diagnosis-median values and $1-3$ quartiles.

\begin{tabular}{lcccccc}
\hline & Control group & Sepsis group & ELGAN group & NEC group & Rotavirus group & AWD group \\
\hline$N$ & 14 & 12 & 24 & 12 & 16 & 3 \\
Gender (F/M) & $6 / 8$ & $2 / 10$ & $8 / 16$ & $8 / 4$ & $5 / 11$ & $2 / 1$ \\
Birth weight $(\mathrm{g})$ & $3260(2850-3440)$ & $3208(2505-3465)$ & $1120^{* * *}(800-1400)$ & $1955^{* * *}(1100-2470)$ & $3000(2495-3690)$ & $2250(2100-2700)$ \\
Body weight $(\mathrm{g})$ & $3260(2850-3400)$ & $3208(2505-3465)$ & $1120^{* * *}(800-1400)$ & $1962^{* * *}(1115-2585)$ & $3302(2790-3805)$ & $2270(2190-2610)$ \\
l'Apgar (pts) & $9(8-10)$ & $9(7-10)$ & $5^{* * *}(3-6)$ & $6(5-8)$ & $10(8-10)$ & $6(2-8)$ \\
5'Apgar (pts) & $10(9-10)$ & $10(7-10)$ & $5^{* * *}(4-7)$ & $8(7-8)$ & $10(9-10)$ & $7(7-8)$ \\
GA (wks) & $39(37-39)$ & $38(36-40)$ & $28^{* * *}(25-31)$ & $33^{* * *}(29-36)$ & $38(36-39)$ & $37(36-38)$ \\
DOL & $5(2-11)$ & $10(3-18)$ & $2(1-5)$ & $7(4-25)$ & $24^{* * *}(17-27)$ & $3(2-5)$ \\
MOD (SD/CS) & $9 / 5$ & $8 / 4$ & $6 / 18$ & $3 / 9$ & $7 / 9$ & $0 / 3$ \\
Zonulin (ng/ml) & $3.5(2.7-4.8)$ & $3.5(2.8-4.3)$ & $3.8(2.0-5.4)$ & $8.6(3.8-11.9)$ & $36.0^{* * *}(26.0-43.2)$ & $20.3^{*}(17.7-28.2)$ \\
NTISS (pts) & $3(2-5)$ & $18^{* * *}(13-26)$ & $24^{* * *}(21-26)$ & $23^{* * *}(18-25)$ & $8(7-9)$ & $21^{* *}(19-23)$ \\
CRP $(\mathrm{mg} / \mathrm{dl})$ & $0.2(0.2-1.6)$ & $52.1^{* * *}(15.8-94.1)$ & $3.1(0.9-9.5)$ & $22.8^{*}(6.1-59.1)$ & $0.7(0.2-2.1)$ & $47.5(1.9-58.2)$ \\
PCT $(\mathrm{ng} / \mathrm{ml})$ & $0.1(0.1-0.2)$ & $9.0^{* *}(2.2-14.2)$ & $5.7^{*}(2.7-10.6)$ & $2.2(0.7-12.0)$ & $0.1(0.1-0.3)$ & $2.1(0.9-3.3)$ \\
WBC $\left({ }^{*} 1000 / \mu \mathrm{l}\right)$ & $11.7(9.9-13.0)$ & $10.8(5.7-21.0)$ & $16.6^{*}(9.6-23.1)$ & $13.3(11.3-19.8)$ & $10.8(8.1-115)$ & $10.3(7.6-24.2)$ \\
PLT $\left({ }^{*} 1000 / \mu l\right)$ & $277(185-322)$ & $174(86-258)$ & $133^{* *}(92-187)$ & $226(166-359)$ & $386^{* *}(310-484)$ & $230(213-263)$ \\
\hline
\end{tabular}

ELGAN: extremely low gestational age newborn; NEC: necrotizing enterocolitis; AWD: abdominal wall defect; N: number; F: female; M: male; g: grams; pts: points; GA: gestational age; wks: weeks; DOL: day of life; MOD: mode of delivery; SD: spontaneous delivery; CS: cesarean section. Statistical significance versus control group. ${ }^{*} p<0.05 ;{ }^{* *} p<0.01 ;{ }^{* * *} p<0.001$.

\section{Results}

Demographic and clinical characteristics of the study population are presented in Table 1 separately for each group of patients. Significantly lower body weight at the time of evaluation and gestational age values were seen in the NEC and ELGAN groups; the last one was also described by lower 1st and 5th minute Apgar score, as could be expected. Newborns suffering from rotavirus infection were significantly older ( 24 and 17-25 days of life, median and $1-3 \mathrm{Q}$, resp.). Median CRP values were highest in the group of term newborns with sepsis and in patients with NEC (52.1 (1-3Q: 15.8-94.1) and 22.8 (1-3Q: 6.1-59.1) $\mathrm{mg} / \mathrm{dl}$, resp.), while PCT values were also elevated in septic newborns and in extremely low gestational age babies (9.0 (1-3Q: 2.2-14.2) and 5.7 (1-3Q: 2.7-10.6) $\mathrm{ng} / \mathrm{ml}$, resp.). We cannot exclude that the elevation of PCT in the group of premature babies was due to the physiological rise in PCT in the first 48 hours of life. Decrease in platelet count was noticeable in the ELGAN group with median $133 \times 10^{3} / \mu 1$, but not in NEC patients, in whom this pathology was more frequently expected.

Analysis of serum $\mathrm{ZO}$ concentration revealed statistically higher median values in the rotavirus infection group and gastroschisis patients (36.0 (1-3Q: 26.0-43.2) and 20.3 (13Q:17.7-28.2) $\mathrm{ng} / \mathrm{ml}$, resp.). Median ZO concentration in the NEC group was more than twice as high as in controls, but the difference did not reach statistical significance due to the low number of study cases.

We could not find any correlation between $\mathrm{ZO}$ values and parameters of inflammatory response (CRP, PCT) (Table 2). A low platelet number as well as some demographic parameters (1st and 5th minute Apgar score, body weight) correlated with $\mathrm{ZO}$ concentration (Table 2).
TABLE 2: Correlation coefficients (by Spearman) between zonulin levels and selected demographic and clinical parameters.

\begin{tabular}{lcc}
\hline & R (Spearman) & $p$ value \\
\hline WBC & -0.215 & NS \\
PLT & 0.448 & $<0.001$ \\
NTISS & -0.186 & NS \\
CRP & -0.100 & NS \\
PCT & -0.101 & NS \\
GA & 0.196 & NS \\
1'Apgar & 0.313 & 0.007 \\
5'Apgar & 0.289 & 0.01 \\
BW & 0.316 & 0.004 \\
\hline
\end{tabular}

WBC: white blood count; PLT: platelets; NTISS: Neonatal Therapeutic Intervention Scoring System; CRP: C-reactive protein; PCT: procalcitonin; GA: gestational age; BW: body weight.

\section{Discussion}

Our results suggest that $\mathrm{ZO}$ is a valuable biomarker of intestinal injury. We showed that $\mathrm{ZO}$ concentration is markedly increased in newborns with rotavirus infection and to a less extent in patients with gastroschisis and those suffering from NEC. The study data consist of an indirect evidence for increased intestinal permeability. Certainly, rotavirus infection is a clinical model of massive injury to the intestinal epithelium that has to be followed by increased permeability [16]. Clinically interesting, but unanswered question is whether an injury to TJs, pictured by elevated ZO serum concentration, could be considered as a biomarker of risk for intestinal bacterial translocation even without perforation. Our rotavirus infection group, despite showing the highest 
values of $\mathrm{ZO}$, did not present severe deterioration of clinical condition in septic mechanism. We could not find any published paper on $\mathrm{ZO}$ and rotavirus infection, but there was one regarding enteroviruses. Vorobjova et al. [17] reported correlations between an intensity of mucosal atrophy in small bowels and increased $\mathrm{ZO}$ serum levels (comparable to $\mathrm{ZO}$ concentrations in our rotavirus group) with a higher density of enteroviruses.

We checked the course of disease in the individuals representing the NEC group and found that $\mathrm{ZO}$ values were not higher in patients with perforation. Therefore, we suggest that increased ZO level reflects more the extent (area) than the severity of intestinal injury. We would not however dare to draw any strong conclusions, as the study group of patients with NEC was small and our project was not designed as a prospective observation. Nevertheless, the idea of a new potential biomarker of intestinal wall injury seems to be very promising.

A search of medical databases is providing just few papers on the topic of ZO in paediatric population. Recently, a mild increase in $\mathrm{ZO}$ and proinflammatory cytokines was revealed in infant colic, that is, suspected to be at least partially caused by low-grade systemic inflammation [18].

A problem of microinflammatory injury to the intestinal barrier is raised in patients with type 2 diabetes and obesity $[10,19]$. Much more severe intestinal injury occurs in patients with Crohn's disease [6]. In all these entities, elevated $\mathrm{ZO}$ was detected along with increased inflammatory markers (CRP, PCT, IL6, and TNF- $\alpha$ among others). Therefore, increased $\mathrm{ZO}$ level may possibly be considered as another inflammatory parameter. However, in our study, we showed no correlation between $\mathrm{ZO}$ and proinflammatory markers. We cannot deny yet that longer observation may have changed this relation. An alternative explanation for our findings is that $\mathrm{ZO}$ level reflects more interstitial injury than inflammation generated by the injury. This interesting hypothesis necessitates further studies.

Observations made in NEC patients characterised by relatively low values of $\mathrm{ZO}$ in comparison to newborns with rotavirus infection are in line with our suggestions. NEC patients showed high NTISS punctuation and markedly elevated CRP and PCT, which all together fit in a clinical picture of severely ill newborns, mostly premature ones. The group included individuals with perforation and severe sepsis, but ZO concentrations were much lower than those in the rotavirus group. The last ones did not have any indicators of severe inflammation, and the course of disease was stable and uncomplicated.

We are aware that NEC is a multifactorial entity and many components decide on the final clinical presentation. Intestinal microbiota, including the number and diversity of bacterial species, antibiotic usage, feeding regimen, and the kind of nutritional support (human milk versus formula) are important explanatory features in NEC occurrence, severity of disease course, and prognosis [20, 21]. Many positive opinions are stated about probiotics and its protective role against NEC development and severity, possibly by decreasing inflammatory response, including proinflammatory cytokine secretion [15]. Very likely, there are other factors of significant influence on disease outcome, and one of them could be congenital heart malformation causing the decrease in peripheral perfusion, leading to tissue necrosis and NEC as consequences. In such situation, a bacterial causative role in pathogenesis of NEC might be diminished. Also, the marked differences in response to NEC, presented by small intestines and colon, cannot be excluded as meaningful factors in final prognosis [20]. All this emphasized that many aspects need to be taken into consideration in analyzing the relation between $\mathrm{ZO}$ levels and clinical picture of NEC. Our observations were not supported by all the necessary data and the results we attained in NEC patients rise many questions regarding intestinal epithelium and mechanisms of intestinal necrosis, and at this point, conclusions would be premature.

Our data acquired from septic patients again were not quite expected. The development of septic SIRS is strongly related to the gut and dysfunction of the intestinal wall, with a crucial role of epithelial TJs and intestinal permeability, leading to increased proinflammatory activity [4, 22, 23]. This is consistent with the results published by Klaus et al. [24] who measured ZO concentration in adult septic patients and found its values elevated in comparison to nonseptic individuals. However, there were no differences in $\mathrm{ZO}$ levels between septic cases of abdominal origin and those of other ethiopathogenesis. Again, according to Deitch [25] and Faries et al. [26], also burns or trauma were causing an increase in intestinal permeability. These findings would suggest that $\mathrm{ZO}$ release from TJs and intestinal permeability are induced by a "causative" factor(s) stimulating these processes indirectly. Proinflammatory factors seem to be a good explanation. That is why we actually suspected the simultaneous increase in inflammatory parameters (CRP, PCT, and leucocytes) in good correlation with $\mathrm{ZO}$ levels, but our results did not prove it. Similarly, Klaus et al. did not demonstrate any correlation between $\mathrm{ZO}$ and inflammatory markers [24]. Another author observed that perioperative probiotic treatment was beneficial for patients, reducing the rate of infections, decreasing $\mathrm{ZO}$ levels and intestinal permeability (bacterial DNA measurement), and reducing the time of antibiotic treatment [27]. This suggests that the composition of intestinal microflora and possibly the proportions of "good" and "bad" bacteria matters. Unfortunately, we did not have the data regarding intestinal bacterial transfer in sepsis, so we were not able to compare these results. It would also be difficult to discuss the differences in ZO levels, as our septic patients presented rather low values. The reason for this may lie in their general condition, not severe enough to show spectacular rise of the marker. However, to finally evaluate $\mathrm{ZO}$ role in sepsis, we should check its value also in very rare but severe cases, when peripheral perfusion is almost stopped, peristalsis is paralysed and risk of intestinal injury rises significantly. The other thing is to follow $\mathrm{ZO}$ levels for several days during sepsis. Longer observational period may reveal changes in $\mathrm{ZO}$ which were not noticed in one-point measurements.

We did not have many opportunities to compare ZO levels in extremely premature infants with values in other patients. Saleem et al. [21] observed 43 newborns below 33 weeks of gestation, including 12 equal or lower than 28 weeks and 
measured ZO, alpha-1-anti-trypsin, and the urinary lactulose/ rhamnose ratio. They found no correlation between $\mathrm{ZO}$ and the lactulose/rhamnose ratio and concluded that intestinal barrier function was impaired in that group of patients and maturation depended on gestational and postnatal age. What is more, according to Saleem et al., ZO was not involved in TJ maturation. This could be an explanation for our results, but the lack of absolute $\mathrm{ZO}$ values in a cited article withdraws us from making comparisons. Analyzing the ELGAN group, we were not surprised that CRP was not high (frequently seen in less matured newborns) and we analyzed PCT levels with caution, keeping in mind its peak value on the first two days of life. A low number of platelets were in parallel to generally serious clinical condition, confirmed by high NTISS evaluation.

Newborns suffering from gastroschisis created a group with a very small number of patients, and it prevented us from drawing more sophisticated statistical descriptions. Nevertheless, we noticed particularly high ZO concentration, even though the newborns' intestines were not injured by squeezing, torsion, introduction of feeding, or invasive procedure before and at the time of blood sampling.

Talking about $\mathrm{ZO}$ as a marker of intestinal permeability, we did not prove the disturbances in intestinal wall "leakage" by golden standard, which means enteral administration of unabsorbed substance followed by its measurement in urine samples. A mixture of lactulose and rhamnose disaccharides is currently recommended for this test, but evaluation of urine samples is technically demanding [21]. Clinically more important is the fact that prematures suspected for NEC or newborns in septic shock are not allowed to consume any enteral substances, especially when it comes to just about research and not proved medication.

This pilot study indicates the urgent need for further evaluation of intestinal condition in typical neonatal entities and search for marker or panel of markers enabling to identify individuals at risk of bacterial translocation followed by severe sepsis or intestinal perforation.

Summarizing the results, the following conclusions can be proposed:

1. Zonulin is a promising biomarker of intestinal condition, possibly an injury, and is markedly elevated in rotavirus infections in newborns.

2. Its role in defining the severity of necrotizing enterocolitis and risk for perforation is not well described and needs further evaluation.

3. An increase in zonulin may not be parallel to the release of inflammatory markers, and low CRP should not exclude an injury to neonatal intestines.

\section{Ethical Approval}

All procedures performed in studies involving human participants were in accordance with the ethical standards of the institutional research committee and with the 1964 Helsinki Declaration and its later amendments or comparable ethical standards. Study protocol was approved by the Ethics Committee of Medical University of Silesia [KNW/0022/KB1/120/11/13/14; KNW/0022/ $\mathrm{KB} 1 / 121 / 11 / 13]$, and the consent was issued for the use of remained biological material.

\section{Conflicts of Interest}

The authors declare that there is no conflict of interest regarding the publication of this paper.

\section{Acknowledgments}

This study was supported by the grants from Medical University of Silesia (KNW-1- 077/P/1/0, KNW-1-057/P/2/0, and $\mathrm{KNW}-003 / \mathrm{N} / 7 / \mathrm{K})$.

\section{References}

[1] B. Baudry, A. Fasano, J. Ketley, and J. B. Kaper, "Cloning of gene (zot) encoding a new toxin produced by Vibrio cholera," Infection and Immunity, vol. 60, pp. 428-434, 1992.

[2] A. Fasano, B. Baudry, D. W. Pumplin et al., "Vibrio cholera produces a second enterotoxin, which affects intestinal tight junctions," Proceedings of the National Academy of Sciences of the United States of America, vol. 88, pp. 5242-5246, 1991.

[3] A. Sapone, L. De Magistris, M. Pietzak et al., "Zonulin upregulation is associated with increased gut permeability in subjects with type 1 diabetes and their relatives," Diabetes, vol. 55, pp. 1443-1449, 2006.

[4] M. P. Fink, "Intestinal epithelial hyperpermeability: update on the pathogenesis of gut mucosa barrier dysfunction in critical illness," Current Opinion in Critical Care, vol. 9, pp. 143151, 2003.

[5] S. Tsukita and M. Furuse, "The structure and function of claudins, cell adhesion molecules at tight junctions," Annals of the New York Academy of Sciences, vol. 915, pp. 129-135, 2000.

[6] T. Vanuytsel, S. Vermeire, and I. Cleynen, "The role of Haptoglobin and its related protein, Zonulin, in inflammatory bowel disease," Tissue Barriers, vol. 1, article e27321, 2013.

[7] A. Tripathi, K. M. Lammers, S. Goldblum et al., "Identification of human zonulin, a physiological modulator of tight junctions, as prehaptoglobin-2," Proceedings of the National Academy of Sciences of the United States of America, vol. 106, pp. 16799-16804, 2009.

[8] A. Fasano, "Regulation of intercellular tight junctions by zonula occludens toxin and its eukaryotic analogue zonulin," Annals of the New York Academy of Sciences, vol. 915, pp. 214-222, 2000.

[9] W. Wang, S. Uzzau, S. E. Goldblum, and A. Fasano, "Human zonulin, a potential modulator of intestinal tight junctions," Journal of Cell Science, vol. 113, Part 24, pp. 4435-4440, 2000.

[10] B. Jayashree, Y. S. Bibin, D. Prabhu et al., "Increased circulatory levels of lipopolysaccharide (LPS) and zonulin signify novel biomarkers of proinflammation in patients with type 2 diabetes," Molecular and Cellular Biochemistry, vol. 388, pp. 203-210, 2014.

[11] A. Fasano, T. Not, W. Wang et al., "Zonulin, a newly discovered modulator of intestinal permeability, and its expression in celiac disease," Lancet, vol. 355, pp. 1518-1519, 2000.

[12] S. Drago, R. El Asmar, M. Di Pierro et al., "Gliadin, zonulin and gut permeability: effects on celiac and non-celiac intestinal 
mucosa and intestinal cell lines," Scandinavian Journal of Gastroenterology, vol. 41, pp. 408-419, 2006.

[13] J. M. Moreno-Navarrete, M. Sabater, F. Ortega, W. Ricart, and J. M. Fernandez-Real, "Circulating zonulin, a marker of intestinal permeability, is increased in association with obesity-associated insulin resistance," PLoS One, vol. 7, article e37160, 2012.

[14] T. Watts, I. Berti, A. Sapone et al., "Role of intestinal tight junction modulator zonulin in the pathogenesis of type 1 diabetes in $\mathrm{BB}$ diabetic prone rats," Proceedings of the National Academy of Sciences of the United States of America, vol. 102, pp. 2916-2921, 2005.

[15] X. Ling, P. Linglong, D. Weixia, and W. Hong, "Protective effects of Bifidobacterium on intestinal barrier function in LPS-induced enterocyte barrier injury of Caco-2 monolayers and in a rat NEC model," PLoS One, vol. 11, no. 8, article e0161635, 2016.

[16] J. A. Guttman and B. B. Finlay, "Tight junctions as targets of infectious agents," Biochimica et Biophysica Acta, vol. 1788, no. 4, pp. 832-841, 2009.

[17] T. Vorobjova, H. Raikkerus, L. Kadaja et al., "Circulating zonulin correlates with density of enteroviruses and tolerogenic dendritic cells in the small bowel mucosa of celiac disease patients," Digestive Diseases and Sciences, vol. 62, no. 2, pp. 358-371, 2017.

[18] A. Pärtty, M. Kalliomäki, S. Salminen, and E. Isolauri, "Infantile colic is associated with low-grade systemic inflammation," Journal of Pediatric Gastroenterology \& Nutrition, vol. 64, no. 5, pp. 691-695, 2017.

[19] A. Żak-Gołąb, P. Kocełak, M. Aptekorz et al., "Gut microbiota, microinflammation, metabolic profile, and zonulin concentration in obese and normal weight subjects," International Journal Endocrinology, vol. 2013, Article ID 674106, 9 pages, 2013.

[20] P. Jiang, B. Smith, N. Qvist et al., "Intestinal proteome changes during infant necrotizing enterocolitis," Pediatric Research, vol. 73, no. 3, pp. 268-276, 2013.

[21] B. Saleem, A. C. Okogbule-Wonodi, A. Fasano et al., "Intestinal barrier maturation in very low birthweight infants: relationship to feeding and antibiotic exposure," The Journal of Pediatrics, vol. 183, article e1, pp. 31-36, 2017.

[22] C. L. Leaphart and J. J. Tepas, "The gut is a motor of organ system dysfunction," Surgery, vol. 141, pp. 563-569, 2007.

[23] J. A. Clark and C. M. Coopersmith, "Intestinal crosstalk: anew paradigm for understanding the gut as the "motor" of critical illness," Shock, vol. 28, pp. 384-393, 2007.

[24] D. A. Klaus, M. C. Motal, U. Burger-Klepp et al., "Increased plasma zonulin in patients with sepsis," Biochemical Medicine, vol. 23, no. 1, pp. 107-111, 2013.

[25] E. A. Deitch, "Intestinal permeability is increased in burn patients shortly after injury," Surgery, vol. 107, pp. 411-416, 1990.

[26] P. L. Faries, R. J. Simon, A. T. Martella, M. J. Lee, and G. W. Machiedo, "Intestinal permeability correlates with severity of injury in trauma patients," Journal of Trauma and Acute Care Surgery, vol. 44, no. 6, pp. 1031-1036, 1998.

[27] Z. H. Liu, M. J. Huang, X. W. Zhang et al., "The effects of perioperative probiotic treatment on serum zonulin concentration and subsequent postoperative infectious complications after colorectal cancer surgery: a double-center and double-blind randomized clinical trial," The American Journal of Clinical Nutrition, vol. 97, no. 1, pp. 117-126, 2013. 


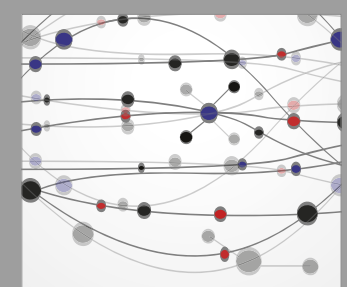

The Scientific World Journal
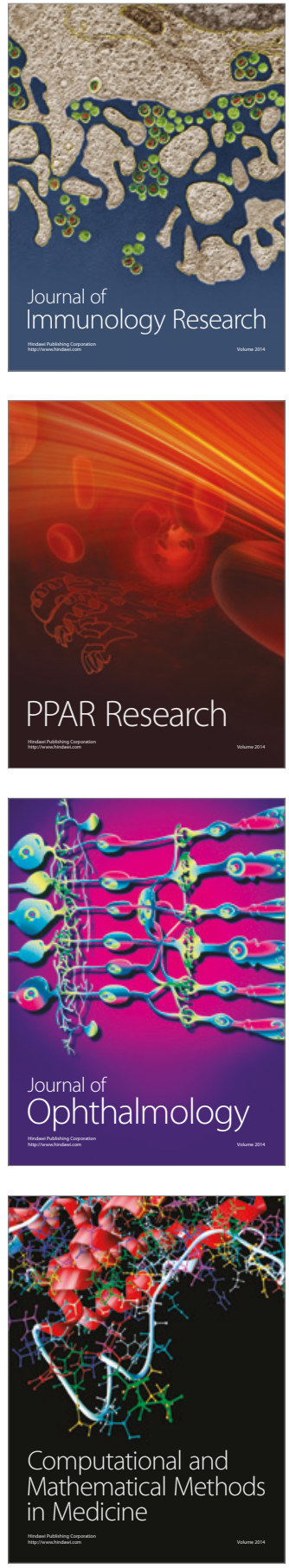

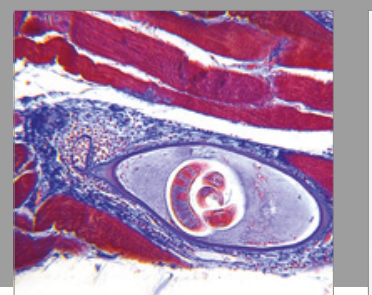

Gastroenterology Research and Practice
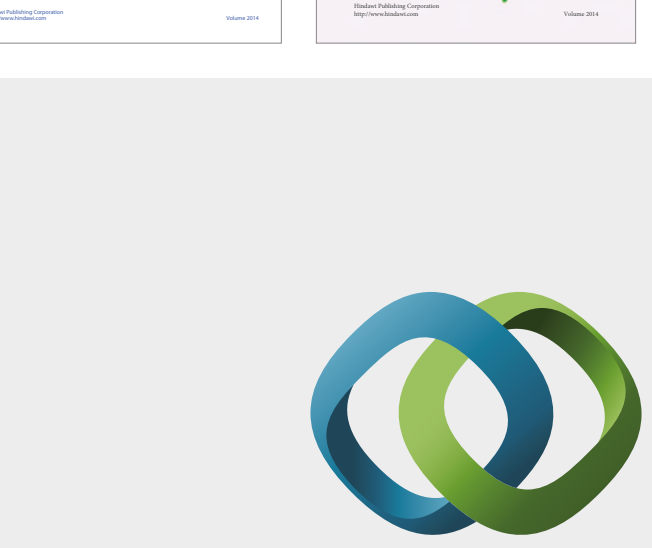

\section{Hindawi}

Submit your manuscripts at

https://www.hindawi.com
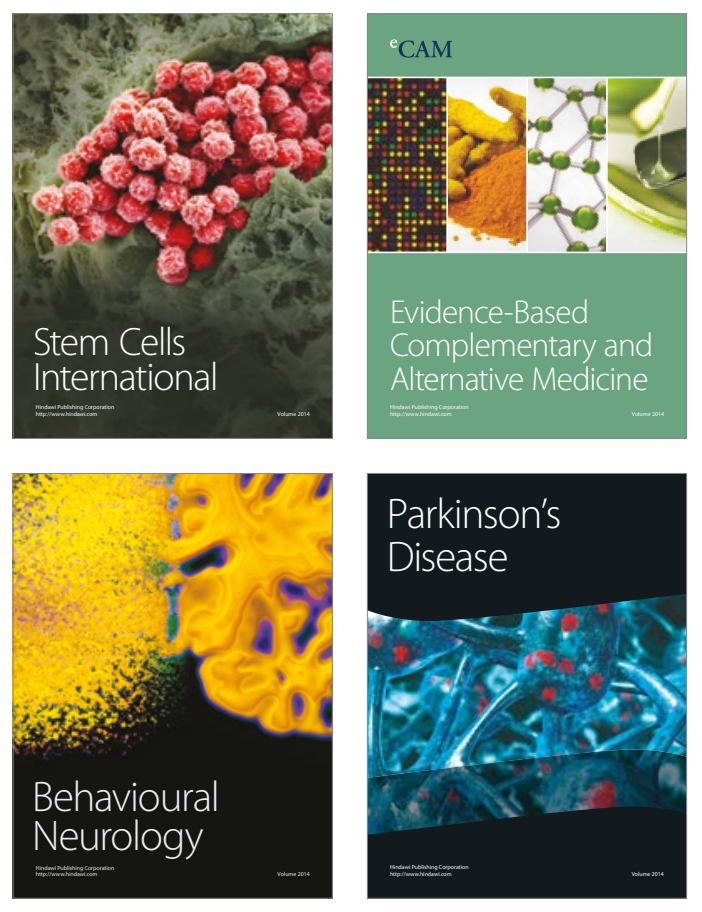
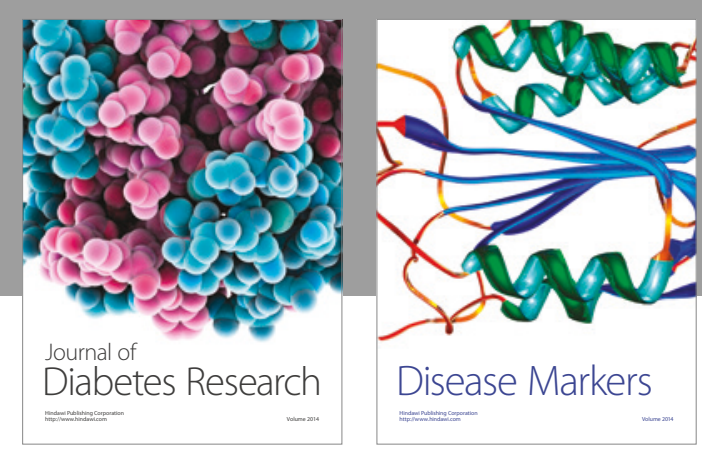

Disease Markers
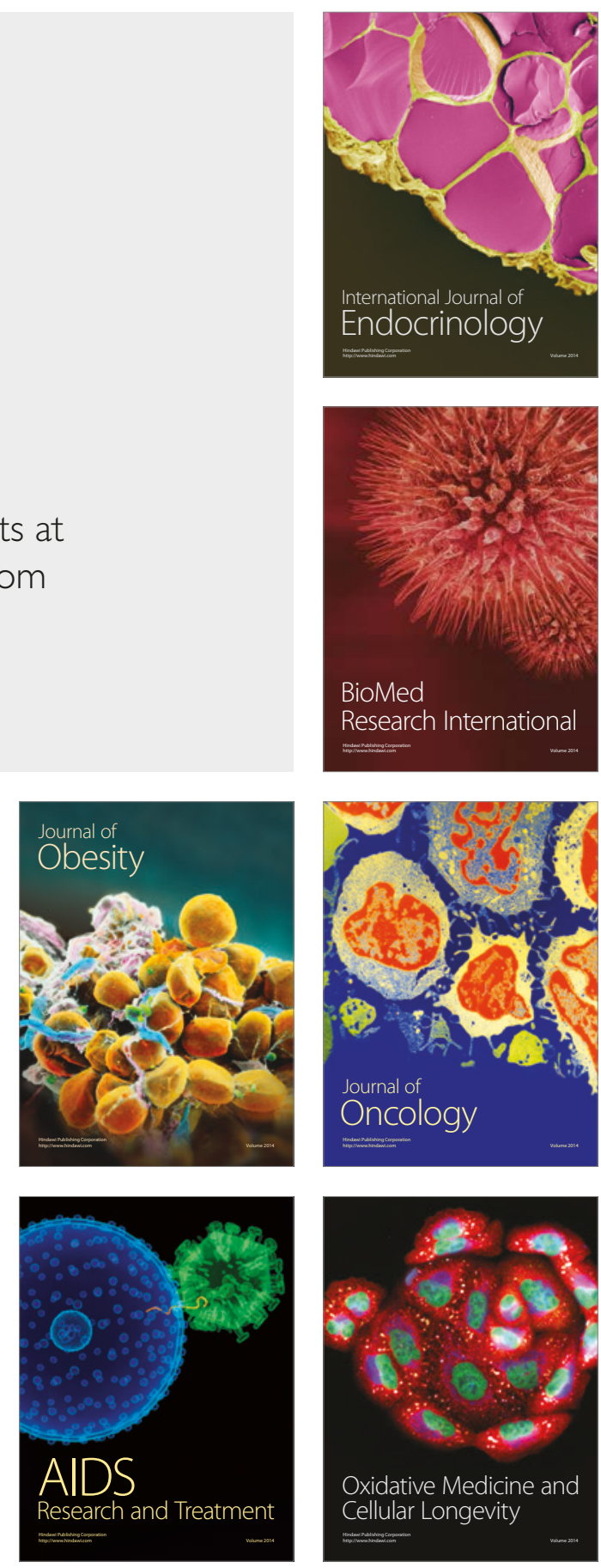